University of Nebraska - Lincoln

DigitalCommons@University of Nebraska - Lincoln

\title{
Optimal Segment Size for Fixed-sized Segment Protection in Wavelength-routed Optical Networks
}

\author{
Raghunath Tewari \\ University of Nebraska-Lincoln, rtewari@cse.unl.edu \\ Byrav Ramamurthy \\ University of Nebraska-Lincoln, bramamurthy2@unl.edu
}

Follow this and additional works at: https://digitalcommons.unl.edu/cseconfwork

Part of the Computer Sciences Commons

Tewari, Raghunath and Ramamurthy, Byrav, "Optimal Segment Size for Fixed-sized Segment Protection in Wavelength-routed Optical Networks" (2009). CSE Conference and Workshop Papers. 94.

https://digitalcommons.unl.edu/cseconfwork/94

This Article is brought to you for free and open access by the Computer Science and Engineering, Department of at DigitalCommons@University of Nebraska - Lincoln. It has been accepted for inclusion in CSE Conference and Workshop Papers by an authorized administrator of DigitalCommons@University of Nebraska - Lincoln. 


\section{Optimal Segment Size for Fixed-sized Segment Protection in Wavelength-routed Optical Networks}

\author{
Raghunath Tewari \\ Dept. of Computer Science and Engineering \\ University of Nebraska - Lincoln \\ Lincoln, NE 68588 \\ Email: rtewari@cse.unl.edu
}

\author{
Byrav Ramamurthy \\ Dept. of Computer Science and Engineering \\ University of Nebraska - Lincoln \\ Lincoln, NE 68588 \\ Email: byrav@cse.unl.edu
}

\begin{abstract}
Protecting a network against link failures is a major challenge faced by network operators. The protection scheme has to address two important objectives - fast recovery and minimizing the amount of backup resources needed. Every protection algorithm is a tradeoff between these two objectives.

In this paper, we study the problem of segment protection. In particular, we investigate what is the optimal segment size that obtains the best tradeoff between the time taken for recovery and minimizing the bandwidth used by the backup segments. We focus on the uniform fixed-length segment protection method, where each primary path is divided into fixed-length segments, with the exception of the last segment in the path. We observe that the optimal segment size for a given network depends on several factors such as the topology and the ratio of the costs involved.
\end{abstract}

\section{INTRODUCTION}

Link failures in wavelength-routed optical networks is a well studied problem [1], [2]. There can be several reasons due to which a link might fail, such as fiber cuts, aging of components, local failures and natural calamities. In these cases, it becomes necessary to have a mechanism to either repair the link or route the traffic along a different path. Each of the two methods has its own advantages and disadvantages. Repairing the link does not require any backup paths to be setup in the network but on the downside, it involves time. For time-sensitive data this might not be a feasible option.

Various algorithms have been proposed to protect network traffic from disruptions due to link failures. Broadly they can be divided into three categories - link protection, path protection and segment protection. The idea of link protection is to set up backup paths for each link in the network. If a link fails, then the backup path is used to route the traffic. In path protection there is a backup path from the source to the destination. If any link in this path fails, the failure information is relayed back to the source, which then sends the data via the backup path. Segment protection is a generalization of the previous two concepts. The path from source to destination is partitioned into several segments. Each segment has exactly one backup path. If a link failure occurs in one of the segments, this information is relayed back to the source of the segment, which then routes the data via the backup path of that segment. Thus we can see that segment protection is a generalization of the other two kinds of protection methods: a segment size of 1 corresponds to link protection and a segment size equal to the path length corresponds to path protection. Segment protection is also called sub-path protection in the literature. Various forms of segment protection have been proposed and studied [3], [4], [5], [6], [7], [8].

A classification of the various protection schemes has been proposed in [7]. According to that classification, the protection problem that we consider here would fit into the uniform, fixed partitioning scheme with sub-path replacement. The main advantage of considering fixed (sub-path) segment size is that it simplifies the design and operation and thereby results in reduced complexity and cost. As networks become larger in scale, it is important to look for simple mechanisms for failure recovery which have only a small impact on the network management and design complexities.

\section{RELATED WORK}

Algorithms for segment protection have been developed and studied in earlier works. In [6] the authors develop a shared segment protection scheme called PROMISE that combines path and link protection to obtain two approaches to solve the problem. Their first contribution is an ILP formulation to find segments that optimize the total cost incurred due to time delay and bandwidth used. They allow for overlapping active segments. This formulation is not feasible for large networks. They also develop a polynomial time heuristic that gives a near-optimal set of segments and their corresponding backups for a given active path when compared to the ILP formulation. The downside of this implementation is the complexity of the algorithm that creates the backup paths. Since there are an exponential number of sub-paths that needs to be considered, in the worst case the algorithm takes exponential time to compute the backup paths.

In another work [7], the authors present a dynamic partitioning sub-path protection routing technique for routing a Fault Tolerant Path Set (FTPS) in a network. Their algorithm partitions the primary path into sub-paths and then dynamically computes their backup paths. Similar to our study, they also consider the case when only a single link failure occurs. Their algorithm is dynamic in nature, in the sense that, when a connection request arrives, a path is searched for from the source to the destination with the necessary bandwidth. If this 
fails, then the connection is rejected. Else, wavelengths are assigned along this path setting up a primary path. When a link fails, the traffic is routed on to an already reserved backup path. The authors propose a dynamic segment protection method which takes into account current network conditions (such as wavelength availability) in identifying the segments.

In this paper, we focus on the uniform fixed-length segment protection method under static network conditions, where each primary path is divided into fixed-length segments, with the exception of the last segment in the path. If the path length is $p$ and the segment size chosen is $k$, there will be $\lfloor p / k\rfloor$ segments of length $k$ and, optionally, a final segment of length $p-\lfloor p / k\rfloor \times k$. For a fixed-length segment protection method, the segment size $(k)$, once determined, would remain the same for all the primary paths of connections set up in the network. Therefore, deciding an optimal segment size for a network is of very high importance. This is because, such an optimal segment size would lower the cost (as defined in the next section) associated with link failures. However, it is not evident how to solve this problem. One reason for this is that we do not even know what factors might affect the optimal segment size for a given network. In this study, we investigate some of the factors that we think might affect the optimal segment length and study their influence on the optimal segment length.

\section{PROBLEM DESCRIPTION}

Link failures can be of several types. For example there can be multiple links that fail simultaneously, there can be a partial failure of some link, etc. In this project, we study networks with a single link failure.

Given a source and a destination in a network, we look at the shortest path from the source to the destination. We consider a partition of this path into several segments. Now if a link in this path fails, that (failure) information needs to be conveyed to the the first node of the segment in which that link lies. We call this node, the source of that segment. This source node then routes the data via the backup path of that segment. It is entirely possible that the segment does not have a backup path. In this case, there is no other alternative but to repair the failed link, which is often a time-consuming process.

We now formalize this model as follows. Let $G=(V, E)$ be a network(graph) where $V$ is the set of nodes (vertices) and $E$ is the set of links (edges) in $G$. Fix a source and a destination, $s, t \in V$ respectively. Let $p=\left(x_{0}, x_{1}, \ldots, x_{n}\right)$ be the path from $s$ to $t$. Let $P=\left(p_{1}, p_{2}, \ldots, p_{k}\right)$ be a partition of $p$ into segments with $P^{\prime}=\left(p_{1}^{\prime}, p_{2}^{\prime}, \ldots, p_{k}^{\prime}\right)$ being their respective backups. Let $e=(x, y)$ be a link that fails in $p$. Let $e$ belong to $p_{i}$ for some $i$ between 1 and $k$. The information of the failed link gets conveyed to the source of the segment $p_{i}$, which then resends the data via $p_{i}^{\prime}$. Denote the path from the source of the segment $i$ to the link $e$ by $p_{i_{e}}$. Let $\alpha$ be the cost per unit distance of transmitting the failure notification back to the source of a segment. Let $\beta$ be the cost per unit distance of reserving a link as part of a backup path. Let $\gamma$ be the cost per unit length of repairing a failed link. Denote weight of a path ( or link) as $w(p)$ (or $w(e)$ ). Then the cost associated with the failure of a link $e$ is,

$$
c(e)=\min \left\{\gamma w(e), \alpha w\left(p_{i_{e}}\right)+\beta w\left(p_{i}^{\prime}\right)\right\} .
$$

The goal of this study is to come up with a segment size that minimizes

$$
\frac{\sum_{e \in E} c(e)}{|E|},
$$

and to investigate the effects of the parameters $\alpha, \beta$ and $\gamma$ in minimizing this value.

\section{IMPLEMENTATION AND EXPERIMENT}

We randomly generate a graph of varying sizes and varying "edge probability". That is, for $0 \leq c \leq 1$, the edge probability, and for every edge position, we place an edge with probability $c$. Next we fix a source and a sink and and use Dijkstra's algorithm [9] to find a shortest path between them.

For every edge in the path, we calculate of cost of rerouting traffic, in case there is a failure, and take the average across all paths. We do this for every possible partition of the path. Although there are exponentially many such partitions, since our path length is typically about 10 , we can afford the exponential run time. We thus determine the optimal segment size, that is for what average segment size is the cost minimized.

As we mentioned earlier, our setup has three parameters, $\alpha, \beta$ and $\gamma . \alpha$ and $\beta$ are constants that affect the rerouting costs while $\gamma$ is the constant cost for link repairing. At this point we would like to emphasize that the parameter $\gamma$ has been introduced for the completion of the model. Typically we would want $\gamma$ to be sufficiently high, corresponding to the fact that, whenever possible, rerouting should be done. We fix $\gamma=\infty$.

The entire implementation is done in Matlab. Fig 1 shows the flowchart for the implementation.

\section{REsults}

We perform two sets of experiments. In the first set, we fix the size of the graphs to be 1000 . Next we generate a random graph of size 1000 and edge probability of 0.1 and 0.15 . For each of these graphs we find the optimal segment size averaged and rounded off over all pairs of source and destination, as we vary the ratio $\alpha / \beta$. The results are summarized in Fig. 2 .

Thus we see that as $\alpha$ increases the optimal segment size decreases. In other words, as the cost of sending information of failure to the source node increases, the segment size decreases. The curves are not monotonic though. This is because of the fact that the graphs constructed are random and there might be other factors that affect the segment size as well.

In another set of experiments we investigated the effect of the size of the graph on the optimal segment size. We fix $c=0.15, \alpha / \beta=1$ and vary the size of the graphs from 200 to 2000 in steps of 200 . The results are shown in Fig. 3.

As we can see from the figure, there is no definite correlation between the size of the graph and the optimal segment 


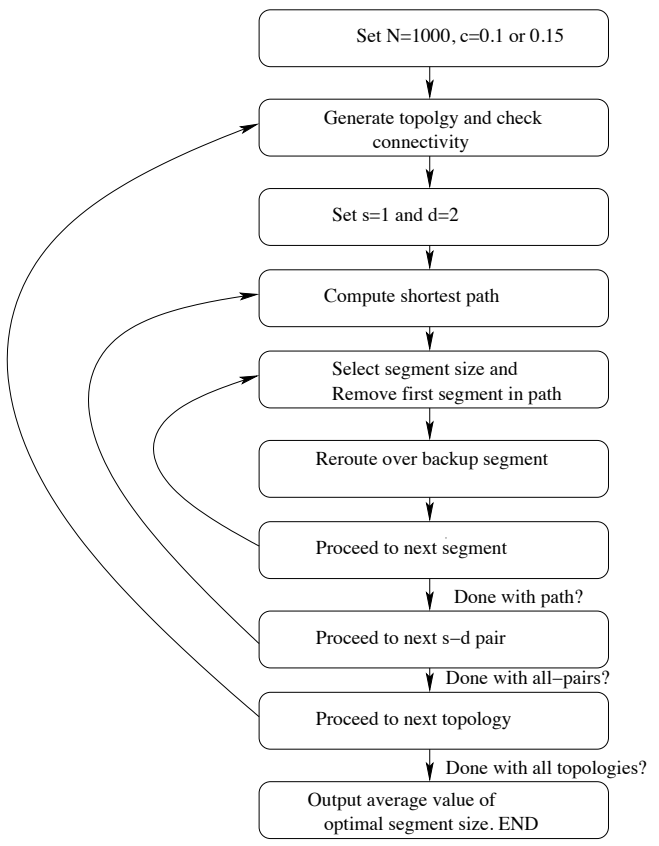

Fig. 1. Flowchart describing the implementation in Matlab.

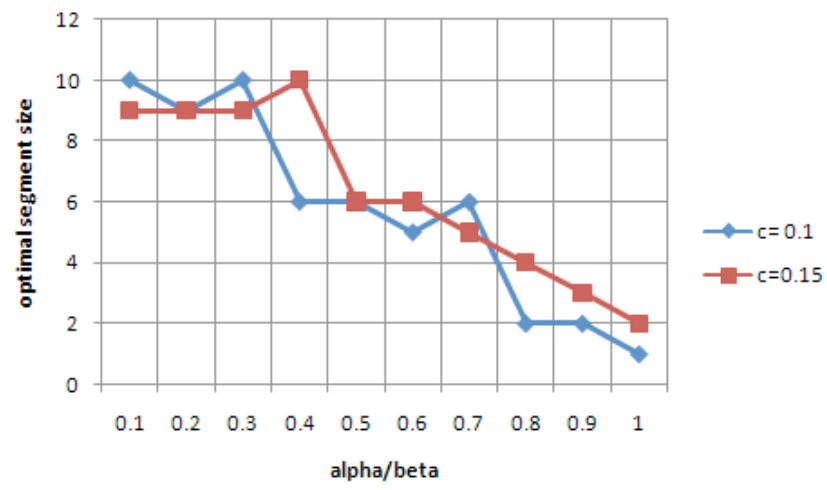

Fig. 2. Optimal segment size as a function of the ratio of $\alpha / \beta$.

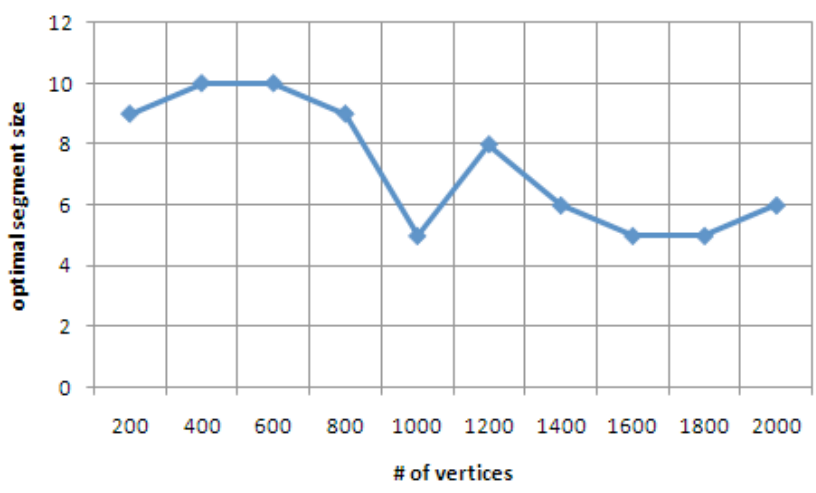

Fig. 3. Optimal segment size as a function of the graph size. size. However for smaller graphs the fluctuation is much higher. As the size of the graph keeps increasing, the optimal size tends to have smaller variations.

In another experiment we tried to observe the effect of the density of the graph on its optimal size. This experiment was not very fruitful in the sense that, for $c=0.2$, the path between the source and destination was too short to observe any significant optimal segment size. For $c<0.1$, the graphs were quite sparse and most often a backup path did not exist. For this reason we decided to perform all our experiments with the values $c=0.1$ and $c=0.15$.

\section{CONCLUSION AND Future WORK}

Thus we see that in a wavelength-routed network, the topology of the network as well the ratio of the cost of transmitting the failure information back to the source to that of reserving a backup path, plays a crucial role in deciding the optimal segment size. This gives us a way to decide the optimal segment size, irrespective of the traffic through the network.

There is a lot of scope for future work in this area. The correlation that we observe in our experimental results is not very high. Can we get a better correlation by taking into account more factors? Also, what happens if the model changes? That is, what happens if there are multiple edge failures or there are computation costs involved at nodes? Are there any other graph theoretic properties (such as degree, number of cycles, minimum cut size) that might affect the problem? These questions are very interesting and need careful investigation.

\section{REFERENCES}

[1] M. Tornatore, M. Carcagní, C. S. Ou, B. Mukherjee, and A. Pattavina, "Intelligent shared-segment protection," Comput. Netw., vol. 52, no. 10, pp. 1965-1974, 2008.

[2] X. Wang, L. Guo, X. Wei, L. Pang, T. Wu, J. Du, and X. Wang, "A new algorithm with segment protection and load balancing for singlelink failure in multicasting survivable networks," in Journal of Systems and Software, 2008.

[3] P. Ho and H. Mouftah, "A framework for service guaranteed shared protection in wdm mesh networks," in IEEE Communications Magazine, vol. 23, pp. 97-103, 2002.

[4] C. Ou, S. Rai, and B. Mukherjee, "Extension of segment protection for bandwidth efficiency and differentiated quality of protection in optical/mpls networks," in Optical Switching and Networking, pp. 19-33, 2005.

[5] P. Ho and H. Mouftah, "A novel survivable routing algorithm for segment shared protection in mesh wdm networks with partial wavelength conversion," in IEEE Journal of Selected Areas on Communications, vol. 22, pp. 1539-1548, 2004.

[6] D. Xu, Y. Xiong, and C. Qiao, "Novel algorithms for shared segment protection," in IEEE Journal on Selected Areas in Communications, vol. 21, 2003.

[7] A. Todimala and B. Ramamurthy, "A dynamic partitioning sub-path protection routing technique in wdm mesh networks," in Cluster Computing, vol. 7, pp. 259-269, 2004.

[8] K. P. Gummadi, M. J. Pradeep, and C. S. R. Murthy, "An efficient primary-segmented backup scheme for dependable real-time communication in multihop networks," vol. 11, pp. 81-94, Feb 2003.

[9] E. W. Dijkstra, "A note on two problems in connexion with graphs," in Numerische Mathematik, vol. 1, pp. 269-271, 1959. 